\title{
Comparison of surgical resection versus transarterial chemoembolization with additional radiation therapy in patients with hepatocellular carcinoma with portal vein invasion
}

\author{
Jin Wook Chung \\ Department of Radiology, Seoul National University Hospital, Seoul National University College of Medicine, Seoul, Korea
}

Keywords: Hepatocellular carcinoma; Surgical resection; Chemoembolization; External beam radiotherapy

\section{See Article on Page 144}

According to Barcelona clinic liver cancer (BCLC) staging and treatment allocation system, chemoembolization is recommended as the first-line treatment for intermediate-stage hepatocellular carcinoma (HCC) and sorafenib for advanced-stage HCC.' However, recently proposed Hong Kong Liver Cancer staging and treatment allocation system suggests that intermediate HCC (one of the following three factors: larger than $5 \mathrm{~cm}$, more than 3 in number, branch portal vein invasion or hepatic vein invasion) can be indicated for hepatic resection and locally advanced HCC (two of the three factors) can be indicated for chemoembolization. ${ }^{2}$ More recently, there are a growing number of articles reporting better outcome of hepatic resection for HCC in large solitary nodule, intermediate stage, or portal vein invasion when compared with chemoembolization. ${ }^{3}$ However, all of them were retrospective in the study design.
In the current issue, Lee et al. conducted a retrospective study comparing surgical resection and chemoembolization with additional radiotherapy in patients with HCC with portal vein invasion. ${ }^{4}$ They compared the survival outcome of 43 patients who underwent surgical resection for $\mathrm{HCC}$ with portal vein invasion without previous treatment with that of another 43 patients who received chemoembolization followed by radiotherapy as initial treatment. Both groups were matched for Child-Pugh class, tumor size, and extent of portal vein invasion. And, they demonstrated that the cumulative overall survival was significantly longer in patients treated with resection than in those treated with chemoembolization followed by radiotherapy, and surgical resection was an independent predictive factor for better survival outcome on multivariate analysis.

However, because of major problem in the study design, it is difficult to agree with the results of this study. In the resection group, the authors excluded the patients with resection margin involvement and incomplete tumor removal. It means that the

\section{Abbreviations:}

BCLC, Barcelona clinic liver cancer; HCC, hepatocellular carcinoma

\section{Corresponding author : Jin Wook Chung}

Department of Radiology, Seoul National University Hospital, Seoul National University College of Medicine, 101 Daehak-ro, Jongno-gu, Seoul 03080, Korea

Tel: +82-2-2072-2584, Fax: +82-2-743-6385

E-mail: chungjw@snu.ac.kr

http://orcid.org/0000-0002-1090-6872

Received : Jan. 2, 2018/ Accepted : Feb. 5, 2018

Copyright $\odot 2018$ by Korean Association for the Study of the Liver

This is an Open Access article distributed under the terms of the Creative Commons Attribution Non-Commercial License (http://creativecommons.org/licenses/by-nc/3.0/) which permits unrestricted non-commercial use, distribution, and reproduction in any medium, provided the original work is properly cited. 
patients with "surgically resectable" HCC on initial evaluation were screened and patients who underwent "curative resection" were finally selected. As a result, the survival outcome of resection group was extremely good. For fair comparison, the same selection criteria should be applied to chemoembolization plus radiotherapy group. However, unfortunately, it is impossible to do so. In order to solve this problem, the authors should include patients with "surgically resectable" HCC regardless of resection margin involvement and incomplete tumor removal in the resection group and exclude patients with "surgically unresectable" HCC from chemoembolization plus radiotherapy group. Matching with Child-Pugh class, tumor size, and extent of portal vein invasion was not enough. Even after matching, the survival outcome of chemoembolization plus radiotherapy in this study was almost same with that of their previous reports. ${ }^{5,6}$ In determining resectability of HCC, we should consider performance status, hepatic functional reserve, degree of portal hypertension, tumor distribution (localized, unilobar, bilobar), tumor location (central, peripheral), and intrahepatic metastasis as the authors partly pointed out in the discussion section. Those factors should be included in the selection criteria and/or matching process.

I agree with the facts that intermediate and advanced HCCs are really diverse and, therefore, subclassification is needed. There is a chance that more aggressive therapies including hepatic resection may reveal better survival outcome in the favorable subgroups than standard therapies. Because of difficulties conducting prospective randomized trials in HCC, I believe it is still valuable to continue our efforts to find out the subgroups in which hepatic resection shows better outcome than standard therapies from our routine practice cohort. However, for validity of retrospective studies comparing hepatic resection with standard therapies in advanced HCCs, every effort should be made to eliminate or minimize the preexisting huge selection bias because hepatic resection is almost always selected in patients with good hepatic functional reserve, good performance status, no co-morbidities, and localized tumors.

\section{Conflicts of Interest}

The author has no conflicts to disclose.

\section{REFERENCES}

1. Forner $A$, Reig ME, de Lope $C R$, Bruix J. Current strategy for staging and treatment: the BCLC update and future prospects. Semin Liver Dis 2010;30:61-74.

2. Yau T, Tang VY, Yao TJ, Fan ST, Lo CM, Poon RT. Development of Hong Kong Liver Cancer staging system with treatment stratification for patients with hepatocellular carcinoma. Gastroenterology 2014;146:1691-1700.

3. Zhang XP, Wang K, Li N, Zhong CQ, Wei XB, Cheng YQ, et al. Survival benefit of hepatic resection versus transarterial chemoembolization for hepatocellular carcinoma with portal vein tumor thrombus: a systematic review and meta-analysis. BMC Cancer 2017;17:902.

4. Lee DB, Lee HC, An JH, Shim JH, Kim KM, Lim YS, et al. Comparison of surgical resection versus transarterial chemoembolization with additional radiation therapy in patients with hepatocellular carcinoma with portal vein invasion. Clin Mol Hepatol 2018;24:144150.

5. Kim GA, Shim JH, Yoon SM, Jung J, Kim JH, Ryu MH, et al. Comparison of chemoembolization with and without radiation therapy and sorafenib for advanced hepatocellular carcinoma with portal vein tumor thrombosis: a propensity score analysis. J Vasc Interv Radiol 2015;26:320-329.

6. Chung SR, Kim JH, Yoon HK, Ko GY, Gwon DI, Shin JH, et al. Combined cisplatin-based chemoembolization and radiation therapy for hepatocellular carcinoma invading the main portal vein. J Vasc Interv Radiol 2015;26:1130-1138. 